\title{
Changes in Structure, Interstitial Cajal-like Cells and Apoptosis of Smooth Muscle Cells in Congenital Ureteropelvic Junction Obstruction
}

\author{
Mitra Mehrazma1, MD; Parin Tanzifi*2,3, MD; Naser Rakhshani , MD
}

${ }^{1}$ Oncopathology Research Center, ${ }^{4}$ Firouzgar Hospital, Iran University of medical sciences, ${ }^{2}$ Department of Pathology, ${ }^{3}$ Children's Medical Center, Pediatrics Center of Excellence, Tehran University of Medical Sciences, Tehran, Iran

Received: Aug 03, 2013; Accepted: Jan 20, 2014; First Online Available: Jan 31, 2014

\begin{abstract}
Objective: The goal of this study is to evaluate some structural changes in muscular, collagenous and neural components as well as expression of Cajal-like cells and apoptosis of smooth muscle cells in congenital ureteropelvic junction obstruction (UPJO).

Methods: Tissue specimens were obtained from 25 patients with UPJO and compared with normal ureteropelvic junction regions of 19 autopsies. In paraffin embedded sections the amount of Cajal-like cells, density of nerve fibers and smooth muscle cell apoptosis (using immunohistochemical staining) were determined. Collagen deposition and muscular components were stained by Trichrome-Masson staining and evaluated by image analysis techniques. Arrangement of muscular bundles was also evaluated qualitatively.

Findings: The number of Cajal-like cells was significantly lower in patients than in controls. The apoptotic score and mean number of nerve fibers were not statistically different for the two groups. Arrangement of muscular fibers was more irregular in patients than in controls $(P<0.001)$. Collagen deposition was significantly higher in patients than in controls $(P<0.001)$. The mean amount of muscular component was lower in patients than in normal ones. $(P=0.09)$
\end{abstract}

Conclusion: We found significant pathologic changes in congenital ureteropelvic junction obstruction such as decrease in Cajal-like cells, increase in collagen deposition and irregular arrangement of muscle fibers.

Iranian Journal of Pediatrics, Volume 24 (Number 1), February 2014, Pages: 105-110

Key Words: Uretero-Pelvic Junction Stenosis; Image Analysis; Immunohistochemistry; Interstitial Cell of Cajal-Like Cells

\section{Introduction}

Ureteropelvic junction obstruction (UPJO) is the most common cause of congenital obstruction of urinary system with the prevalence of 1 in 1000 to 2000 neonates ${ }^{[1-3]}$. However, the mechanism of this obstruction has not been known well. This has attracted the attention of many investigators. It is now believed that the disease is multifactorial. It seems that the decrease in the number of smooth muscle cells, interstitial Cajal-like cells and nerve fibers in this region together with abnormal arrangement of smooth muscle cells and increase in collagen deposition have roles in the pathogenesis of the disease ${ }^{[4]}$. The number of studies on the role of apoptosis in smooth muscle cells is also limited and has led to different results ${ }^{[5-8]}$.

In the proximal parts of the urinary system, peristaltic contractions result in the outflow of urine from pelvis through the ureter to the bladder[3]. The outset of these contractions results

\footnotetext{
* Corresponding Author;

Address: Children's Medical Center, No 62, Dr Gharib St., Tehran, Iran

E-mail: p.tanzifi@yahoo.com

(C) 2014 by Pediatrics Center of Excellence, Children's Medical Center, Tehran University of Medical Sciences, All rights reserved.
} 
from the spontaneous production of pulse potentials that start in proximal part of calyceal system and adjust by pacemaker tissue ${ }^{[3]}$.

The interstitial cells of Cajal have been known as pacemakers for the contraction activity of gastrointestinal system. Some studies have been done on the role of Cajal-like cells as pacemakers in the upper parts of the urinary system [2,3,5,6,9-15].

The goal of this study was to examine a series of pathologic changes occurring in congenital ureteropelvic junction obstruction. The results can help with the expression of new therapeutic modalities.

\section{Subjects and Methods}

For the patient group, we used tissue specimens from ureteropelvic junction of 25 children with the mean age of 20 months (50 days to 8 years) with congenital ureteropelvic junction obstruction who underwent pyeloplasty. Prior to the surgery, their problem was confirmed by imaging study, and other causes of urinary obstruction were ruled out. Ureteropelvic junction of 19 matched autopsies with no obstruction in this region was used as control.

Paraffin embedded blocks were first prepared from the specimens followed by slide preparation from each specimen and stained by H\&E method and trichrome-masson staining. Immunohistochemical stained slides of S-100 (Polyclonal Rabbit Anti-Human, code N1573 Dako Cytomation, Denmark) for evaluation of nerve fibers, c-kit (Polyclonal Rabbit Anti-Human CD117, code A4502 Dako Cytomation, Denmark) for Cajal-like cells and caspase-3 (CPP32, clone JHM62, Novocastra Laboratories Ltd, Newcastle Upon Tyne, United Kingdom) for apoptotic smooth muscle cells were prepared according to the instructions of the factory in charge of the production. All slides were observed under light microscopic examination. Interstitial Cajal-like cells were counted in the randomly-selected $5 \mathrm{HPF}$ in each group. Since mast cells are also immunoreactive for c-kit, only those cells with large vesicular nuclei, asteroid shape and small perinuclear cytoplasms having two or more slender cytoplasmic processes localized inbetween muscle fibers were counted. Nerve fibers were immunoreactive for S-100 and were counted in 5 HPF(s) too. Due to the cytoplasmic reaction of caspase 3 antibody, the apoptosis of smooth muscle cells was categorized as $(<25 \%$, $25-50 \%, 51-75 \%,>75 \%)$ with the intensity $(0-3+)$ of immunoreaction to the marker. Collagen deposition has blue color in trichrome-masson staining and its amount was measured by computer analysis of 5 randomly selected HPFs in two groups. Muscular component is red in trichrome-masson staining and was quantitatively evaluated like collagen deposition. In image analysis, areas other than ureteral wall were not considered in measurement. Arrangement of muscle fibers was compared qualitatively between the two groups.

In order to perform statistical analysis, the software SPSS-15 was utilized. We used abundance and ratio for qualitative variables and mean and standard deviation for quantitative ones. The comparison of the means and ratios was done by t-test and $\chi^{2}$-test respectively. $P$ values less than 0.05 were considered to be significant.

\section{Findings}

The mean number of interstitial Cajal-like cells was $14.5 \pm 5.6$ per 5 HPFs in patients and 32.8 \pm 11.9 per 5 HPFs in controls, which reveals significant difference between the two groups $(P<0.001)$ (Fig. 1).

The intensity of immunoreactivity of smooth muscle cells to caspase-3 was 0 in $7(28 \%)$ patients, $1+$ in 8 (32\%), $2+$ in 7 (28\%) and $3+$ in 3 (12\%) patients. In the control group, 7 cases (36.8\%) showed the intensity of $0,7(36.8 \%) 1+$, $2(10.5 \%) 2+$, and $3(15.9 \%) 3+(P=0.2)$.

From the standpoint of the extent of immunoreactivity of smooth muscle cells to caspase-3, it was $<25 \%$ in 12 (48\%) patients, $25 \%-50 \%$ in 5 (20\%), 51\%-75\% in 4 (16\%) and $>75 \%$ in $4(16 \%)$ cases. In the control group, this was $<25 \%$ in $12(63.1 \%)$ cases, $25 \%-50 \%$ in one (5.3\%), $51 \%-75 \%$ in $4(21 \%)$ and $>75 \%$ in 2 $(10.6 \%)$ cases $(P=0.2)$.

The mean score of immunoreaction to caspase3 (which was calculated by multiple intensity 


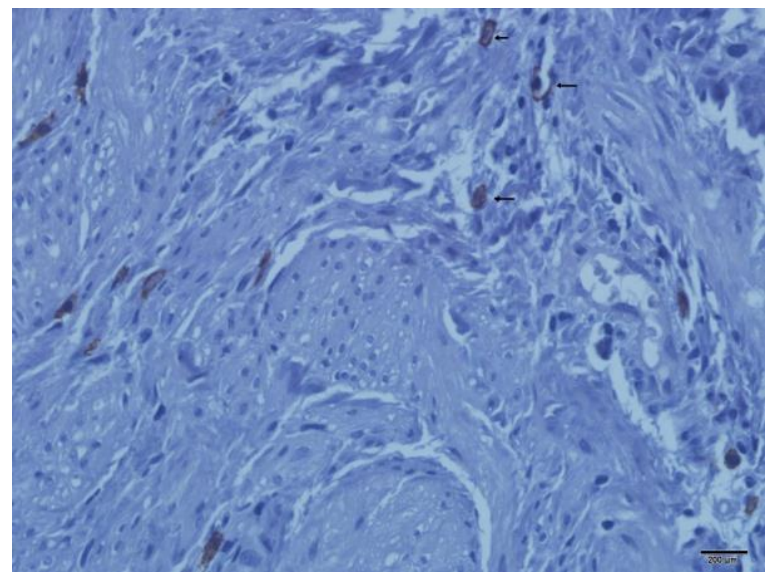

Fig. 1: Cajal-like cells in a control specimen. Note to Cajal-like cells with 2 cytoplasmic processes in between muscle fibers in left side. There are also few mast cells in right side of the image (arrow). (x400)

extent of immunoreaction) in patients was $3.5 \pm 3.1$ and in controls $2.8 \pm 2.5$. There was no significant difference between the two groups $(P=0.4)$ (Fig. 2).

The mean number of nerve fibers in patients was $8.7 \pm 5.2$ per 5 HPFs and $11.4 \pm 8.3$ per 5 HPFs in controls, which shows no significant difference between the two groups $(P=0.2)$.

The arrangement of muscular fibers in 8 (32\%) patients was regular and in 17 (68\%) irregular compared to $17(84.2 \%)$ regular and $2(15.8 \%)$ irregular cases in the control group. Irregular arrangement refers to disorganization and sometimes haphazard arrangement of smooth muscle fibers with deposition of collagen fibers inbetween. Regular arrangement, however, refers to normal array of smooth muscle fibers in this region with inner circular and outer longitudinal layer of smooth muscle fibers. The difference was significant $(P<0.001)$.

The mean amount of collagen deposition in trichrome staining was $156000 \pm 82000 \mathrm{pX1} 1^{2}$ per 5 HPFs in patients and $17800 \pm 6400 \mathrm{pX} 1^{2}$ per 5 HPFs in controls, this shows a significant difference between the two groups $(P<0.001)$ (Fig. 3).

The mean amount of muscular component in 5 randomly-selected HPFs in patients was $331200 \pm 94000$ in comparison with $463100 \pm$ 93000 in the controls which has no meaningful difference $(P=0.09)$. Summary of the results are shown in Table 1.

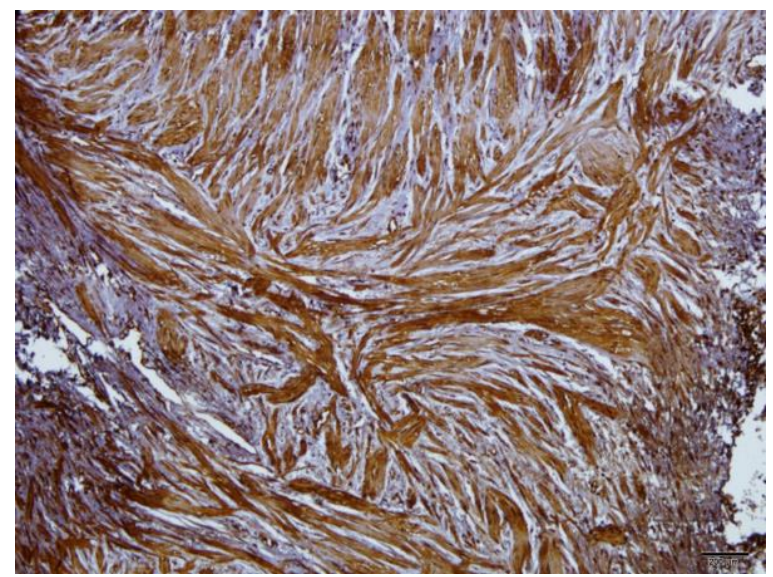

Fig. 2: Immunoreaction of smooth muscle cells to caspase 3 antibody in a patient specimen.(intensified $3+)$ note also to the irregular arrangement of the muscle fibers. $(\times 100)$

\section{Discussion}

The results of this study reveal that the mean number of interstitial Cajal-like cells in patients has significant statistical difference with that of the control group. Similar to the previous studies[3,8,9], it shows that the decrease of these cells may have a role in the pathogenesis of the disease. This is in contrast with Koleda et al in which the number of interstitial Cajal-like cells sparse fields was lower in UPJO group than in controls ${ }^{[10]}$. They concluded that this issue would be probably due to the low mean age of their

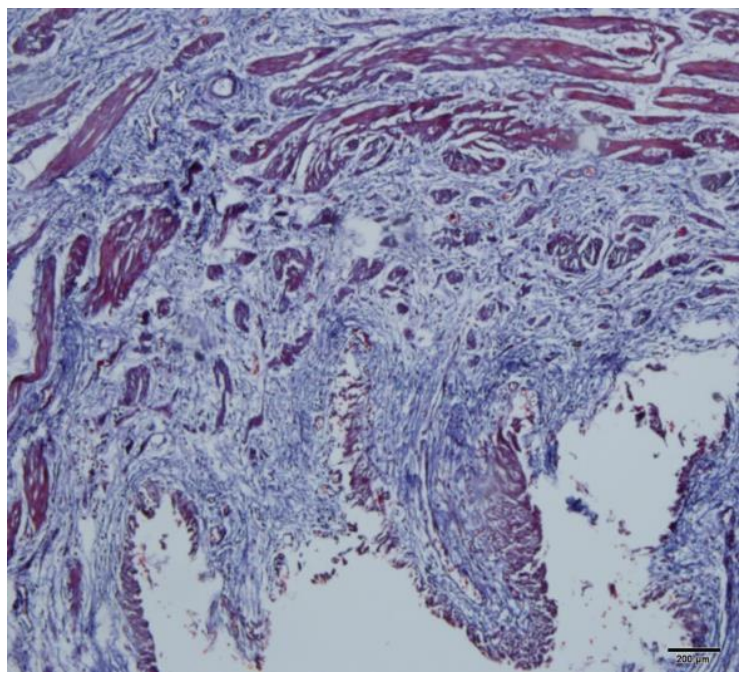

Fig. 3: Increase in collagen deposition in a patient specimen. (Trichrome-Masson stain) $(\times 100)$ 
Table 1: Comparison of results in patients with ureteropelvic junction obstruction and normal cases

\begin{tabular}{|c|c|c|c|}
\hline Variable & Patients & Controls & $P$. value \\
\hline Mean number of Cajal-like cells per 5 HPFs & $14.5(5.6)$ & $32.8(11.9)$ & $<0.001$ \\
\hline $\begin{array}{l}\text { Mean score of immunoreactivity of smooth muscle } \\
\text { cells for caspase-3 }\end{array}$ & $3.5(3.1)$ & $2.8(2.5)$ & 0.4 \\
\hline Mean number of nerve fibers per 5 HPFs & $8.7(5.2)$ & $11.4(8.3)$ & 0.2 \\
\hline Percentage of irregular arrangement of muscle fibers & $68 \%$ & $15.8 \%$ & $<0.001$ \\
\hline Mean amount of collagen deposition per 5 HPFs(pxl² $)$ & $156000(82000)$ & $17800(6400)$ & $<0.001$ \\
\hline Mean amount of muscular component per $5 \mathrm{HPFs}\left(\mathrm{pxl}^{2}\right)$ & $331200(94000)$ & $463100(93000)$ & 0.09 \\
\hline
\end{tabular}

patients and appears as a primary compensatory effect. Surprisingly, the mean age of our patients was also low (20 months) which compares well with theirs (2.3 years). We couldn't rationalize this difference; however, it may be due to the limited number of their controls. Further studies with proper sample volume are needed in this regard to clear the subject.

In this observation we did not find any significant difference for intensity, extent and total score of apoptotic smooth muscle cells between the groups of patients and controls. We found a few studies in the literature about smooth muscle cell apoptosis in UPJO. Kajbafzadeh et al[1] showed that in UPJO there is an increase in smooth muscle cell apoptosis; nevertheless, they evaluated it with TUNEL assay, the method about which there are some criticisms in the literature [16-18]. Arena et al[2] also observed the increase in the apoptosis of smooth muscle cells by using active caspase 3 and western blotting in UPJO specimens, but Özel et al[5] couldn't find any difference between patients and controls in number of apoptotic smooth muscle cells albeit they used Bax antibody for the detection of apoptosis. On the other hand, they found increase in the expression of $\mathrm{Bcl} 2$, an antiapoptotic marker, they related this to the nonspecific inflammation observed in their UPJO specimens. Here, two points should be considered: although there was no statistically significant difference, there were clinically some differences in intensity and overall apoptotic score in patients than in controls. Maybe with increase in the statistical population significant difference will appear. Because of the differences between the present data and a few studies on this subject more research is necessary to clarify the fact.

The mean number of nerve fibers was lower in the patients group than in controls, but it was not statistically significant. With increase in the number of cases, it is probable to see significant difference between the two groups. Evidences from previous projects are more in favor of decrease in neural elements in patients suffering from UPJO[1,4].

We found a clear difference in the arrangement of muscular fibers between patients and controls that is almost irregular in the first group while being regular in the other. This shows that although in some patients decrease in the density of muscular component or apoptosis of smooth muscle cells is not obvious, consideration of the arrangement of muscular component can be helpful in confirmation of the disease. Besides, it may be due to the low mean age of the patients that we could not find meaningful results for the amount of muscular component, because with progression of the disease these structural changes probably will get more severe if they were actually present. Furthermore, we can conclude that irregular arrangement of muscular component may have a role in the pathogenesis of the congenital ureteropelvic junction obstruction, because it has not been known which of these changes occur earlier or later in comparison with each other in the pathologic process; more investigations are needed in this regard.

Our findings showed that the amount of collagen deposition in patients was more than in controls. This might be the result of atrophic changes due to cellular degeneration and substitution of extracellular matrix instead.

There are considerable similarities between the present study and its predecessors. Özel et al found that in all specimens received from patients with UPJO, under light microscopic examination, there were nonspecific evidences of inflammation, epithelial proliferation, atrophy of smooth muscle fibers and fibrosis in the region of stenosis. Fibronectin, collagen type IV and laminin in the 
obstruction site were significantly more than in ureteropelvic regions of normal group. They concluded that significant increase of fibronectin, collagen type IV and laminin in the region of stenosis is one of the pathologic processes in congenital ureteropelvic junction obstruction[5]. Murakumo et al showed that muscle fibers became thinner in patients. Additionally, intercellular spaces became wider, collagen fibers increased significantly, and nerve fibers were less than in controls. They concluded that atrophic changes in muscle fibers and increase in fibrosis are important processes in the development of the disease ${ }^{[4]}$.

We had some limitations in our research. Because of positive immunoreactivity of mast cells to c-kit antibody, it would have been better to check Cajal-like cells with another marker or staining method in addition. However, with respect to morphology of these two cell types and considering the location of Cajal-like cells (inner border of circular muscle layer), the probability of any mistake is low, specifically when we found significant difference between the two groups in immunoreaction to c-kit antibody. Furthermore, it is believed that in fibrotic areas number of mast cells is increased. So, with the assumption of happening the mistake being inevitable, the result would be in favor of increasing the number of c-kit positive cells in patient group (due to increase in mast cells) and therefore we expected this supposed mistake resulting in lower difference rate between the two groups.

\section{Conclusion}

Our study reveals notable pathologic changes in the stenotic site of ureteropelvic junction in children suffering from congenital ureteropelvic junction obstruction. These changes include decrease in interstitial Cajal-like cells, irregular arrangement of muscle fibers and increase in collagen deposition.

\section{Acknowledgment}

This article is the result of a residency thesis and the study was financially supported by a grant from Iran University of Medical Sciences.

\section{Authors' Contribution}

M. Mehrazma: Study design, Critical revision of the manuscript, Funds collection

P. Tanzifi: Concept/Design, Acquisition of data, data analysis and interpretation, preparing the draft of the manuscript N. Rakhshani: Acquisition of data, Critical revision of the manuscript, Study supervision

All authors approved final version of the article.

Conflict of Interest: None

\section{References}

1. Kajbafzadeh AM, Payabvash S, Salmasi AH, et al. Smooth muscle cell apoptosis and defective neural development in congenital ureteropelvic junction obstruction. J Urol 2006;176(2):718-23.

2. Arena F, Nicotina PA, Arena S, et al: C-kit positive interstitial cells of Cajal network in primary obstructive megaureter. Minerva Pediatr 2007; 59(1):7-11.

3. Solari V, Piotrowska AP, Puri P. Altered expression of interstitial cells of Cajal in congenital ureteropelvic junction obstruction. J Urol 2003;170 (6 pt 1):2420-2.

4. Murakumo $M$, Nonomura $K$, Yamashita $T$, et al. Structural changes of collagen components and diminution of nerves in congenital ureteropelvic junction obstruction. J Urol 1997:157(5):1963-8.

5. Özel SK, Emir H, Dervişoğlu S, et al. The roles of extracellular matrix proteins, apoptosis and c-kit positive cells in the pathogenesis of ureteropelvic junction obstruction. J Pediatr Urol 2010;6(2):125-9.

6. Truong LD, Choi YJ, Tsao CC, et al. Renal cell apoptosis in chronic obstructive uropathy: the roles of caspases. Kidney Int 2001;60(1):924-34.

7. Chuang Y, Chuang W, Huang C. Myocyte apoptosis in the pathogenesis of ureteral damage in rats with obstructive uropathy. Urology 2001;58(3):463-70.

8. Cutroneo G, Arena S, Anastasi G, et al. Altered cytoskeletal structure of smooth muscle cells in ureteropelvic junction obstruction. J Urol 2011; 185(6):2314-9.

9. Yang X, Zhang Y, Hu J. The expression of Cajal cells at the obstruction site of congenital pelviureteric junction obstruction and quantitative image analysis. J Pediatr Surg 2009;44(12):2339-42.

10. Koleda P, Apoznanski W, Wozniak Z, et al. Changes in interstitial cell of Cajal-like cells density in congenital ureteropelvic junction obstruction. Int Urol Nephrol 2012;44(1):7-12.

11. Metzger R, Schuster T, Till H, et al. Cajal-like cells in the upper urinary tract. J Urol 2004;172(2):769-72. 
12. Brading AF, McCloskey KD. Mechanisms of Disease: Specialized interstitial cells of the urinary tract - an assessment of current knowledge. Nat Clin Pract Urol 2005;2(11):546-54.

13. Lang RJ, Tonta MA, Zoltkowski BZ, et al. Pyeloureteric peristalsis: Role of atypical smooth muscle cells and interstitial cells of Cajal-like cells as pacemakers. J Physiol 2006;576(Pt 3):695-705.

14. McHale NG, Hollywood MA, Sergeant GP, et al. Organization and function of ICC in the urinary tract. J Physiol 2006;576(Pt 3):689-94.

15. Kang HJ, Lee HY, Jin MH, et al. Decreased interstitial cells of Cajal-like cells possible cause of congenital refluxing megaureters: histopathologic differences in refluxing and obstructive megaureters. Urology 2009;74(2):318-23.
16. Duan WR, Garner DS, Williams SD, et al. Comparison of immunohistochemistry for activated caspase-3 and cleaved cytokeratin 18 with the TUNEL method for quantification of apoptosis in histological sections of PC-3 subcutaneous xenografts. J Pathol 2003;199(2):221-8.

17. Bressenot A, Zimmer O, Marchal S, et al. Detection of apoptosis in vivo: Comparison of different methods in histological sections of subcutaneous xenografts of HT29 human colon adenocarcinoma. Ann Pathol 2009;29(5):370-5.

18. Saraste A. Morphologic criteria and detection of apoptosis. Herz 1999;24(3):189-95. 